\title{
Hyperthermic Intraperitoneal Chemotherapy in Ovarian Cancer
}

\author{
W.J. van Driel, S.N. Koole, K. Sikorska, J.H. Schagen van Leeuwen, \\ H.W.R. Schreuder, R.H.M. Hermans, I.H.J.T. de Hingh, J. van der Velden, \\ H.J. Arts, L.F.A.G. Massuger, A.G.J. Aalbers, V.J. Verwaal, J.M. Kieffer, \\ K.K. Van de Vijver, H. van Tinteren, N.K. Aaronson, and G.S. Sonke
}

The authors' full names, academic degrees, and affiliations are listed in the Appendix. Address reprint requests to $\mathrm{Dr}$. van Driel at the Department of Gynecology, U1-14 the Netherlands Cancer Institute, 1006 BE Amsterdam, the Netherlands, or atw.v.driel@nki.nl.

N Engl J Med 2018;378:230-40. DOI: $10.1056 /$ NEJMoal708618 Copyright @ 2018 Massachusetts Medical Society.

\section{ABSTRACT}

\section{BACKGROUND}

Treatment of newly diagnosed advanced-stage ovarian cancer typically involves cytoreductive surgery and systemic chemotherapy. We conducted a trial to investigate whether the addition of hyperthermic intraperitoneal chemotherapy (HIPEC) to interval cytoreductive surgery would improve outcomes among patients who were receiving neoadjuvant chemotherapy for stage III epithelial ovarian cancer.

\section{METHODS}

In a multicenter, open-label, phase 3 trial, we randomly assigned 245 patients who had at least stable disease after three cycles of carboplatin (area under the curve of 5 to $6 \mathrm{mg}$ per milliliter per minute) and paclitaxel (175 mg per square meter of body-surface area) to undergo interval cytoreductive surgery either with or without administration of HIPEC with cisplatin (100 mg per square meter). Randomization was performed at the time of surgery in cases in which surgery that would result in no visible disease (complete cytoreduction) or surgery after which one or more residual tumors measuring $10 \mathrm{~mm}$ or less in diameter remain (optimal cytoreduction) was deemed to be feasible. Three additional cycles of carboplatin and paclitaxel were administered postoperatively. The primary end point was recurrence-free survival. Overall survival and the side-effect profile were key secondary end points.

\section{RESULTS}

In the intention-to-treat analysis, events of disease recurrence or death occurred in 110 of the 123 patients (89\%) who underwent cytoreductive surgery without HIPEC (surgery group) and in 99 of the 122 patients (81\%) who underwent cytoreductive surgery with HIPEC (surgery-plus-HIPEC group) (hazard ratio for disease recurrence or death, 0.66 ; $95 \%$ confidence interval $[\mathrm{CI}], 0.50$ to $0.87 ; \mathrm{P}=0.003$ ). The median recurrencefree survival was 10.7 months in the surgery group and 14.2 months in the surgeryplus-HIPEC group. At a median follow-up of 4.7 years, 76 patients $(62 \%)$ in the surgery group and 61 patients $(50 \%)$ in the surgery-plus-HIPEC group had died (hazard ratio, $0.67 ; 95 \% \mathrm{CI}, 0.48$ to $0.94 ; \mathrm{P}=0.02$ ). The median overall survival was 33.9 months in the surgery group and 45.7 months in the surgery-plus-HIPEC group. The percentage of patients who had adverse events of grade 3 or 4 was similar in the two groups ( $25 \%$ in the surgery group and $27 \%$ in the surgery-plus-HIPEC group, $\mathrm{P}=0.76$ ).

\section{CONCLUSIONS}

Among patients with stage III epithelial ovarian cancer, the addition of HIPEC to interval cytoreductive surgery resulted in longer recurrence-free survival and overall survival than surgery alone and did not result in higher rates of side effects. (Funded by the Dutch Cancer Society; ClinicalTrials.gov number, NCT00426257; EudraCT number, 2006-003466-34.) 
VARIAN CANCER IS ASSOCIATED WITH the highest mortality of all gynecologic cancers in the western world. The majority of patients receive a diagnosis of advanced disease that has spread beyond the ovaries to the peritoneal surface. The most effective treatment for advanced disease involves a maximum effort to reduce the tumor burden through surgery followed by six cycles of intravenous chemotherapy with carboplatin and paclitaxel. Alternatively, interval cytoreductive surgery is performed after three cycles of chemotherapy. ${ }^{1-4}$ Intraperitoneal delivery of chemotherapy enhances drug delivery at the peritoneal surface and may improve outcomes by eliminating residual microscopic peritoneal disease more efficiently than intravenous administration of chemotherapy.

Combination treatment with intravenous and intraperitoneal chemotherapy has been shown to prolong overall survival after primary cytoreductive surgery among patients with stage III ovarian cancer. ${ }^{5-7}$ Catheter-related problems, increased demands on the patient, and gastrointestinal and renal side effects have hampered the adoption of this approach in most countries. Delivery of the intraperitoneal chemotherapy at the end of surgery can circumvent most of these drawbacks while maintaining its advantages.

Intraperitoneal chemotherapy during surgery that can be delivered under hyperthermic conditions is termed hyperthermic intraperitoneal chemotherapy (HIPEC). Hyperthermia increases the penetration of chemotherapy at the peritoneal surface and increases the sensitivity of the cancer to chemotherapy by impairing DNA repair. Hyperthermia also induces apoptosis and activates heat-shock proteins that serve as receptors for natural killer cells, inhibits angiogenesis, and has a direct cytotoxic effect by promoting the denaturation of proteins..$^{8-11}$ The addition of HIPEC to interval cytoreductive surgery for the treatment of ovarian cancer is feasible, but efficacy data from randomized trials are lacking. ${ }^{12,13}$

We report the results of a randomized, openlabel, phase 3 trial of interval cytoreductive surgery with or without HIPEC in patients with International Federation of Gynecology and Obstetrics stage III ovarian, fallopian tube, or peritoneal cancer who had at least stable disease after three cycles of neoadjuvant chemotherapy with carboplatin and paclitaxel.
METHODS

\section{TRIAL OVERSIGHT}

The trial was designed by an executive committee that included lead investigators and a statistician. Approval for the trial protocol, which is available with the full text of this article at NEJM.org, was obtained from the relevant institutional review boards. Data were collected by the Netherlands Comprehensive Cancer Organisation. Final data collection and analysis were performed by personnel at the data coordinating center at the Department of Biometrics, the Netherlands Cancer Institute, Amsterdam. The first author wrote the initial draft of the manuscript. All the authors contributed to subsequent revisions of the draft, agreed to submit the manuscript for publication, and vouch for the accuracy and completeness of the data and analyses and for the fidelity of the trial to the protocol. There were no agreements regarding confidentiality between the sponsor and either the authors or the participating institutions.

\section{PATIENTS}

Eligible patients had newly diagnosed stage III epithelial ovarian, fallopian tube, or peritoneal cancer and were referred for neoadjuvant chemotherapy because their abdominal disease was too extensive for primary cytoreductive surgery or because surgery had been performed but was incomplete (i.e., after surgery, one or more residual tumors measuring $>1 \mathrm{~cm}$ in diameter were present). Eligibility criteria also included a World Health Organization performance-status score of 0 to 2 (on a scale of 0 to 5 , with higher numbers indicating decreasing performance), normal blood counts, and adequate renal function. All the patients provided written informed consent before enrollment.

\section{TRIAL DESIGN}

We performed a multicenter, randomized, openlabel, phase 3 trial to assess the efficacy and safety of interval cytoreductive surgery with HIPEC as compared with interval cytoreductive surgery without HIPEC. Patients who had received three cycles of neoadjuvant chemotherapy with carboplatin (area under the curve of 5 to $6 \mathrm{mg}$ per milliliter per minute) and paclitaxel (175 mg per square meter of body-surface area) could be registered in the trial before the interval cytoreductive 
surgery took place. Randomization was performed at the time of surgery in cases in which complete or optimal cytoreduction was anticipated. Complete cytoreductive surgery was defined as surgery that resulted in no visible disease (residual disease classification, R-1), optimal cytoreductive surgery as surgery that resulted in the presence of one or more residual tumors measuring less than $2.5 \mathrm{~mm}$ (R-2a) or 2.5 to $10 \mathrm{~mm}$ in diameter (R-2b), and incomplete cytoreductive surgery as surgery that resulted in the presence of one or more residual lesions measuring more than $10 \mathrm{~mm}$ in diameter. For logistic reasons, at two of the eight participating centers, a diagnostic laparoscopy was performed before surgery to evaluate whether complete or optimal surgery was feasible.

At the time of surgery, patients were randomly assigned, in a 1:1 ratio, to undergo interval cytoreductive surgery either with HIPEC (surgery-plusHIPEC group) or without HIPEC (surgery group). Randomization was performed with the use of a minimization procedure, with stratification according to previous surgery (yes vs. no), the hospital in which the surgery was being performed, and the number of involved regions in the abdominal cavity (0 to 5 vs. 6 to 8).

The trial was conducted at eight hospitals at which medical personnel had experience in administering HIPEC in patients with peritoneal disease from colon cancer or from pseudomyxoma peritonei. HIPEC was administered at the end of the cytoreductive surgical procedure with the use of the open technique (detailed information regarding the procedure can be found in the protocol). In brief, the abdomen was filled with saline that circulated continuously with the use of a roller pump through a heat exchanger. By circulation of the heated saline, an intraabdominal temperature of $40^{\circ} \mathrm{C}\left(104^{\circ} \mathrm{F}\right)$ was maintained. Perfusion with cisplatin at a dose of $100 \mathrm{mg}$ per square meter and at a flow rate of 1 liter per minute was then initiated (with $50 \%$ of the dose perfused initially, $25 \%$ at 30 minutes, and $25 \%$ at 60 minutes). The perfusion volume was adjusted such that the entire abdomen was exposed to the perfusate. The HIPEC procedure took 120 minutes in total, including the 90-minute perfusion period. At the end of the perfusion, drains were used to empty the abdominal cavity as completely as possible. To prevent nephrotoxicity, sodium thiosulphate was administered at the start of perfusion as an intravenous bolus ( $9 \mathrm{~g}$ per square meter in $200 \mathrm{ml}$ ), followed by a continuous infusion (12 $\mathrm{g}$ per square meter in $1000 \mathrm{ml}$ ) over 6 hours. Urine production was maintained at a minimum of $1 \mathrm{ml}$ per kilogram per hour during hyperthermic perfusion and for 3 hours after surgery.

Patients received an additional three cycles of carboplatin and paclitaxel after surgery. During follow-up, physical examinations and measurement of the serum cancer antigen 125 (CA-125) level were repeated every 3 months for 2 years and then every 6 months until 5 years after the completion of chemotherapy. Computed tomography was performed at 1, 6, 12, and 24 months after the last cycle of chemotherapy. Patients completed health-related quality-of-life questionnaires - the European Organisation for Research and Treatment of Cancer (EORTC) Quality of Life Questionnaire-Core 30 (QLQ-C30), Quality of Life Questionnaire-Ovarian Cancer Module (QLQ-OV28), and Quality of Life QuestionnaireColorectal Cancer Module (QLQ-CR38) - within 2 weeks before randomization, before the fourth cycle of chemotherapy, 1 week after completion of chemotherapy, and during follow-up at 3, 6, 9, $12,15,18,21$, and 24 months.

\section{END POINTS}

The primary end point was recurrence-free survival, which was defined as the time from randomization to disease recurrence or progression or death from any cause, whichever occurred first. Disease progression was defined according to Response Evaluation Criteria in Solid Tumors (RECIST), version 1.1, or on the basis of an increase from baseline in the CA-125 level, whichever one of these two criteria was met first, as recommended by the Gynecologic Cancer InterGroup (GCIG) ${ }^{14}$ (see the Supplementary Appendix, available at NEJM.org). Secondary end points included overall survival, the side-effect profile, and health-related quality of life; no correction for multiple testing was performed. Data on recurrence-free survival and overall survival were censored at the date of the last contact for the patients who remained alive and had no evidence of disease. The cutoff date for data was set at March 31, 2017.

\section{STATISTICAL ANALYSIS}

We determined that a sample of 245 patients with sufficient follow-up for observation of 192 events of disease recurrence, disease progression, or death would provide the trial with $80 \%$ power to detect 
$50 \%$ longer median recurrence-free survival (27 months vs. 18 months, with a hazard ratio for disease recurrence, disease progression, or death of 0.67$)^{5}$ in the surgery-plus-HIPEC group than in the surgery group, at an overall two-sided type I error rate of 0.05 . A prespecified interim analysis for efficacy was performed after data from $50 \%$ of the required sample were available. The significance level for the final analysis was set at 0.048 to preserve an overall significance level of 0.05 .

Analyses of recurrence-free and overall survival were based on the intention-to-treat population and were stratified according to previous surgery (yes vs. no), the hospital in which the surgery was being performed, and the number of involved areas in the abdominal cavity. Kaplan-Meier estimates were compared with the use of stratified log-rank tests. Hazard ratios and the corresponding $95 \%$ confidence intervals were estimated with the use of Cox proportional-hazards models. Exploratory analyses of recurrence-free survival and overall survival were prespecified for subgroups defined according to previous surgery (yes vs. no) and number of involved regions of the abdominal cavity and were performed post hoc for subgroups defined according to the patients' age ( $<65$ vs. $\geq 65$ years), tumor histologic type (high-grade serous vs. other), and previous laparoscopy (yes vs. no). Hazard ratios for the subgroup analyses are provided with $99 \%$ confidence intervals. Adverse events were graded according to National Cancer Institute Common Terminology Criteria for Adverse Events, version 4.0. In the safety analysis, we included data from all patients who received the assigned treatment. We used mixed-effects growth-curve modeling to evaluate linear and nonlinear changes from baseline in health-related quality of life over time; this modeling adjusted for nonignorable missing data from quality-of-life questionnaires that were not completed.

\section{RESULTS}

\section{PATIENTS}

During the period from April 2007 through April 2016, a total of 245 women were enrolled at eight participating centers in the Netherlands and Belgium. The minimum number of events required for analysis of the primary end point was reached in April 2016, and efficacy data were updated in March 2017. Information on the enrollment, ran- domization, treatment, and follow-up of the patients is shown in Figure 1. Demographic and baseline disease characteristics and surgical and treatment information for the two trial groups are shown in Table 1.

\section{EFFICACY}

After a median follow-up of 4.7 years, 209 of the 245 patients (85\%) had had an event of disease recurrence or death; 137 of the 245 patients (56\%) had died. In total, $83 \%$ of the recurrences were detected on the basis of imaging, irrespective of whether the patient had an increase from baseline in the CA-125 level, and 17\% were detected on the basis of an increase in the CA-125 level alone. In the intention-to-treat analysis, 110 of the 123 patients (89\%) in the surgery group and 99 of the 122 patients (81\%) in the surgeryplus-HIPEC group had an event of disease recurrence or death (hazard ratio, 0.66; 95\% confidence interval $[\mathrm{CI}], 0.50$ to 0.87 ; stratified $\mathrm{P}=0.003$ ) (Fig. 2A). The median recurrence-free survival was 3.5 months longer in the group that underwent cytoreduction surgery with HIPEC than in the group that underwent surgery alone (14.2 months vs. 10.7 months). The probability of recurrencefree survival at 3 years was $8 \%$ in the surgery group (95\% CI, 4 to 16) and 17\% in the surgeryplus-HIPEC group (95\% CI, 11 to 26). Subgroup analyses of recurrence-free survival (Fig. 3) and overall survival (Fig. S2 in the Supplementary Appendix) showed that the effect of HIPEC was consistent across the levels of prespecified stratification factors and post hoc subgroups.

A total of 76 of the 123 patients (62\%) in the surgery group and 61 of the $122(50 \%)$ patients in the surgery-plus-HIPEC group died (hazard ratio, 0.67 ; $95 \% \mathrm{CI}, 0.48$ to 0.94 ; stratified $\mathrm{P}=0.02$ ) (Fig. 2B). The median overall survival was 33.9 months in the surgery group and 45.7 months in the surgery-plus-HIPEC group. The probability of overall survival at 3 years was $48 \%$ (95\% CI, 39 to 58 ) in the surgery group and $62 \%$ (95\% CI, 54 to 72 ) in the surgery-plus-HIPEC group.

\section{SAFETY AND HEALTH-RELATED QUALITY OF LIFE}

The median duration of surgery was 192 minutes (interquartile range, 153 to 251) in the surgery group and 338 minutes (interquartile range, 299 to 426) in the surgery-plus-HIPEC group. More than $95 \%$ of the patients in each group had at least one adverse event of any grade between random- 


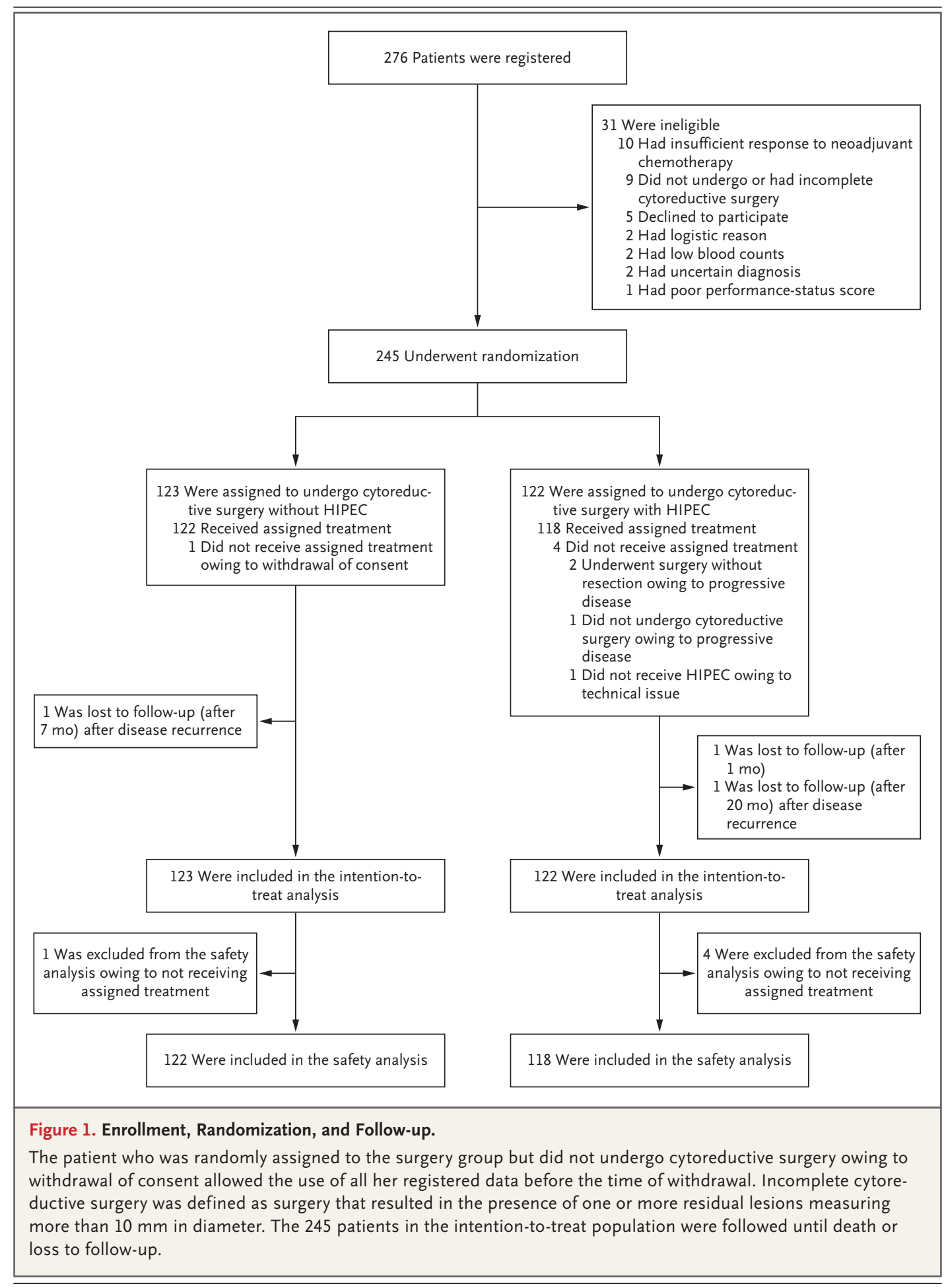

ization and 6 weeks after completion of the last cycle of chemotherapy. No significant differences between the two groups were noted in the incidence of adverse events of any grade. Adverse events of grade 3 or 4 were reported in 30 patients $(25 \%)$ in the surgery group and in 32 patients $(27 \%)$ in the surgery-plus-HIPEC group $(\mathrm{P}=0.76)$. In both groups, the most common events of grade 


\begin{tabular}{|c|c|c|}
\hline Variable & $\begin{array}{l}\text { Surgery } \\
(\mathrm{N}=123)\end{array}$ & $\begin{array}{l}\text { Surgery plus HIPEC } \\
(\mathrm{N}=122)\end{array}$ \\
\hline \multicolumn{3}{|l|}{ Baseline characteristics } \\
\hline Median age (IQR) - yr & $63(56-66)$ & $61(55-66)$ \\
\hline \multicolumn{3}{|l|}{ Tumor histologic type - no. $(\%) \dagger$} \\
\hline High-grade serous & $107(87)$ & $112(92)$ \\
\hline High-grade endometrioid & $1(1)$ & $1(1)$ \\
\hline Carcinosarcoma & $4(3)$ & $1(1)$ \\
\hline Mucinous & $2(2)$ & $1(1)$ \\
\hline Clear-cell carcinoma & $5(4)$ & 0 \\
\hline Low-grade serous & $2(2)$ & $4(3)$ \\
\hline Low-grade endometrioid & 0 & $2(2)$ \\
\hline Metastasis of gastrointestinal tumor & $1(1)$ & 0 \\
\hline Unknown & $1(1)$ & $1(1)$ \\
\hline \multicolumn{3}{|l|}{ Previous surgery — no. (\%) } \\
\hline Yes & $12(10)$ & $12(10)$ \\
\hline No & $111(90)$ & $110(90)$ \\
\hline \multicolumn{3}{|l|}{$\begin{array}{l}\text { Number of regions affected at start of interval cytoreductive } \\
\text { surgery - no. }(\%) \ddagger\end{array}$} \\
\hline $0-5$ & $83(67)$ & $83(68)$ \\
\hline $6-8$ & $40(33)$ & $39(32)$ \\
\hline \multicolumn{3}{|l|}{ Treatment characteristics } \\
\hline \multicolumn{3}{|l|}{ Residual disease after surgery — no. (\%) } \\
\hline R-1, no visible tumor, complete cytoreduction & $82(67)$ & $84(69)$ \\
\hline $\mathrm{R}-2 \mathrm{a}$, tumor nodules $\leq 2.5 \mathrm{~mm}$ & $24(20)$ & $22(18)$ \\
\hline $\mathrm{R}-2 \mathrm{~b}$, tumor nodules $>2.5 \mathrm{~mm}$ and $\leq 10 \mathrm{~mm}$ & $14(11)$ & $13(11)$ \\
\hline Tumor nodules $>10 \mathrm{~mm}$, incomplete cytoreduction & $1(1)$ & 0 \\
\hline No resection』 & $1(1)$ & $2(2)$ \\
\hline No surgery performed & $1(1)$ & $1(1)$ \\
\hline \multicolumn{3}{|l|}{ Bowel resection - no. (\%) } \\
\hline No bowel resection performed & $93(76)$ & $93(76)$ \\
\hline Bowel resection with ileostomy or colostomy & $13(11)$ & $21(17)$ \\
\hline Bowel resection without ileostomy or colostomy & $17(14)$ & $8(7)$ \\
\hline Median duration of surgery (IQR) - min & $192(153-251)$ & $338(299-426)$ \\
\hline Median duration of hospitalization (IQR) — days 9 & $8(7-10)$ & $10(8-12)$ \\
\hline $\begin{array}{l}\text { Median time between surgery and start of first cycle of adju- } \\
\text { vant chemotherapy (IQR) — days }\end{array}$ & $30(25-41)$ & $33(28-41)$ \\
\hline \multicolumn{3}{|l|}{$\begin{array}{l}\text { Number of completed cycles of adjuvant chemotherapy after } \\
\text { surgery - no. (\%) }\end{array}$} \\
\hline 0 & $7(6)$ & $5(4)$ \\
\hline 1 & $2(2)$ & 0 \\
\hline 2 & $3(2)$ & $2(2)$ \\
\hline 3 & $111(90)$ & 115 (94) \\
\hline
\end{tabular}

* There were no significant differences between the trial groups in any of the variables listed in this table, with the exception of the rate of ileostomy or colostomy among the patients who had a bowel resection $(P=0.04)$. Percentages may not sum to 100 because of rounding. HIPEC denotes hyperthermic intraperitoneal chemotherapy, and IQR interquartile range. $\uparrow$ Histologic type was determined on the basis of centrally reviewed pathological assessment.

At the start of surgery, the number of regions involved with disease was assessed as described by Verwaal et al. ${ }^{15}$

$\int$ Surgery was performed, but no resection was possible.

q The median duration of hospitalization included a 1-day stay in the intensive care unit after HIPEC, as specified in the protocol.

The New England Journal of Medicine 


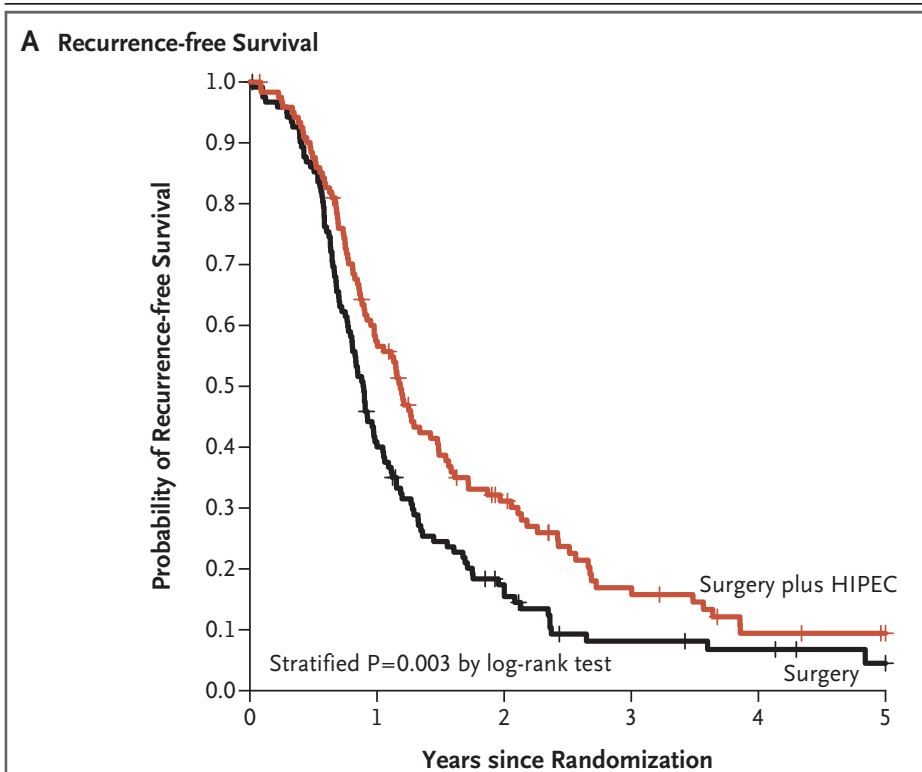

No. at Risk

$\begin{array}{llllrrr}\text { Surgery } & 123 & 48 & 18 & 7 & 5 & 2 \\ \text { Surgery plus } & 122 & 67 & 31 & 15 & 7 & 5\end{array}$

B Overall Survival

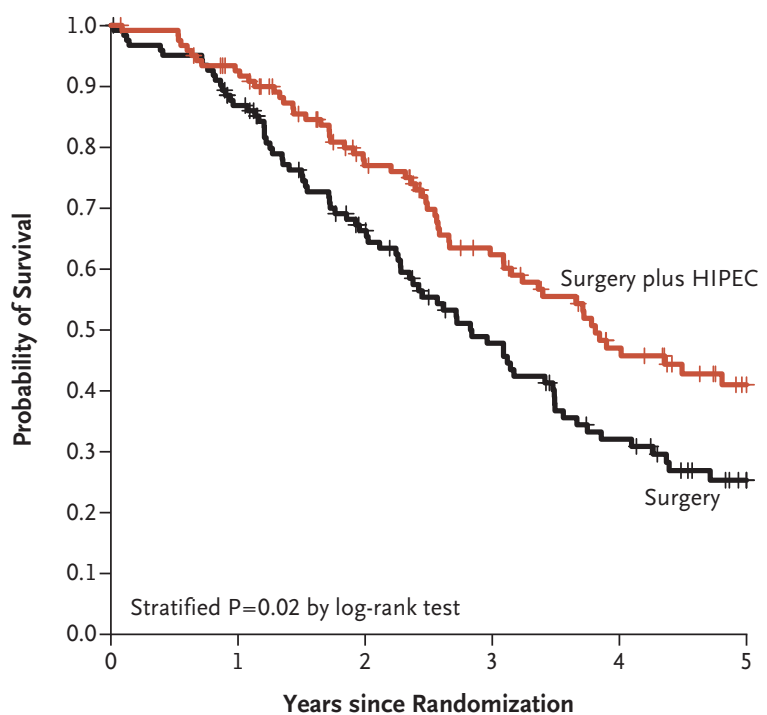

\section{No. at Risk}

$\begin{array}{lllllll}\text { Surgery } & 123 & 103 & 70 & 44 & 27 & 12 \\ \text { Surgery plus } & 122 & 108 & 79 & 56 & 37 & 20\end{array}$

Surgery plus

Figure 2. Kaplan-Meier Estimates of Recurrence-free Survival and Overall Survival.

Panel A shows Kaplan-Meier estimates of recurrence-free survival among patients in the intention-to-treat population. Events of disease recurrence or death were observed in 110 patients $(89 \%)$ in the surgery group and in 99 patients $(81 \%)$ in the surgery-plus-HIPEC group. Panel B shows KaplanMeier estimates of overall survival among patients in the intention-to-treat population. A total of 76 patients (62\%) in the surgery group and $61(50 \%)$ patients in the surgery-plus-HIPEC group died.
3 or 4 were abdominal pain, infection, and ileus (Table 2). One patient in the surgery group died within 30 days after undergoing surgery. A total of 59 patients - 30 in the surgery group and 29 in the surgery-plus-HIPEC group - underwent bowel resection. Among the patients who underwent bowel resection, a colostomy or ileostomy was performed more commonly among patients in the surgery-plus-HIPEC group (21 of 29 patients [72\%]) than among those in the surgery group (13 of 30 patients [43\%]) $(\mathrm{P}=0.04)$. The median total length of hospital admission was 8 days in the surgery group and 10 days in the surgery-plusHIPEC group, including 1 day in the intensive care unit (ICU), as required by the protocol. The median time between the completion of surgery and the restart of chemotherapy after surgery was similar in the two groups - 30 days in the surgery group and 33 days in the surgery-plus-HIPEC group. Rates of completion of all three cycles of chemotherapy after surgery were also similar in the two groups $(90 \%$ and $94 \%$ in the surgery and surgeryplus-HIPEC groups, respectively). A total of 11 patients in the surgery group and 9 in the surgeryplus-HIPEC group had recurrent disease but received no further therapy. We observed no significant differences between the two groups in health-related quality-of-life outcomes over time.

\section{DISCUSSION}

After standard treatment for ovarian cancer, the peritoneal surface is the primary site of disease recurrence. Previous trials that compared six cycles of intraperitoneal chemotherapy plus intravenous chemotherapy with intravenous chemotherapy alone after complete or optimal primary cytoreductive surgery showed that survival was 16 months longer after exposure to chemotherapy at the peritoneal surface than after intravenous chemotherapy alone. ${ }^{5,6,16}$ Nevertheless, the uptake of postoperative intravenous chemotherapy plus intraperitoneal chemotherapy in clinical practice is limited by increased side effects, including catheter-related complications, and the inconvenience of administering therapy intraperitoneally. ${ }^{7,17}$ In the current trial, we evaluated HIPEC as a single administration of intraperitoneal chemotherapy during surgery to overcome the side effects and inconvenience of serial adjuvant intraperitoneal chemotherapy and to improve the distribution of heated chemotherapy in the abdominal cavity. 


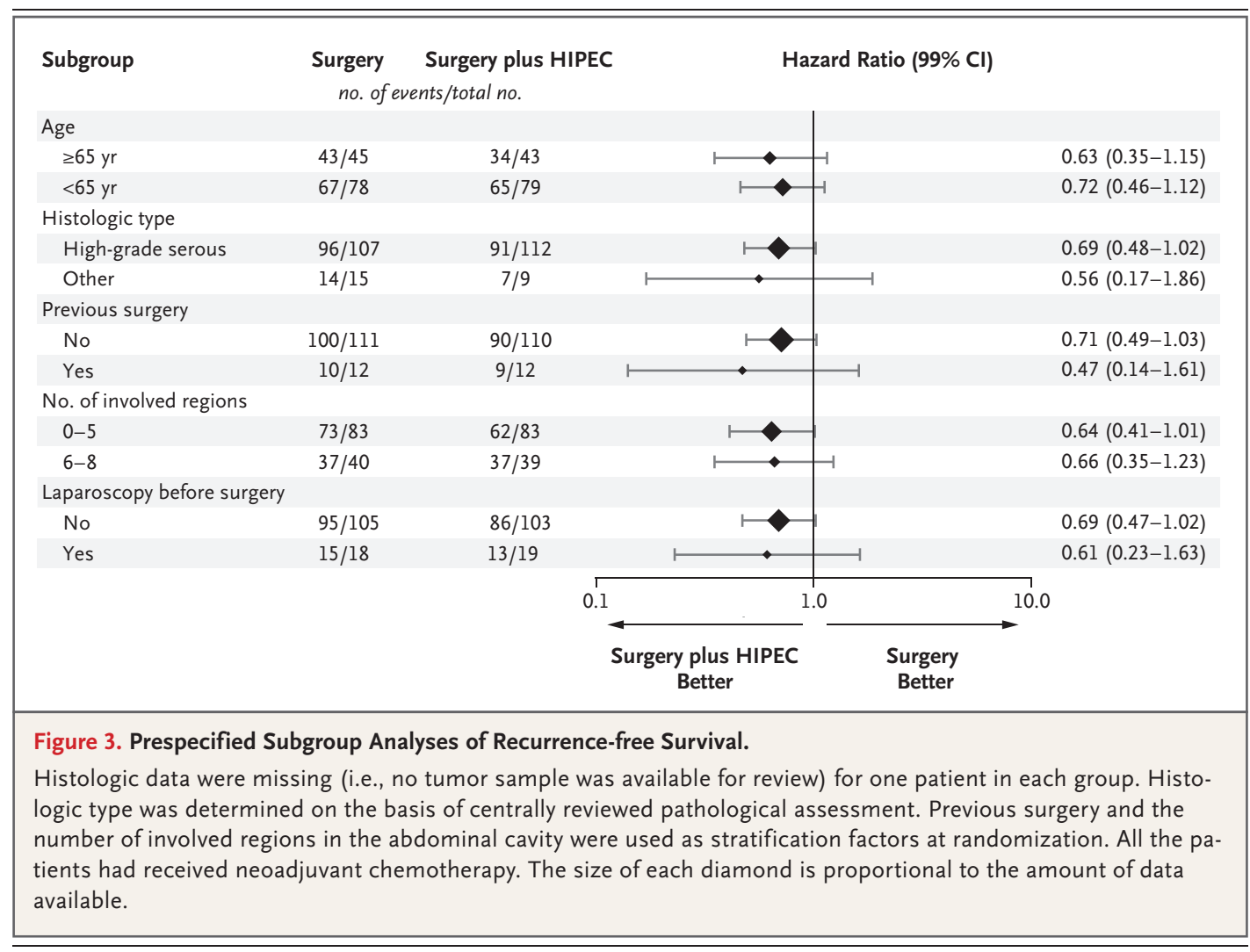

Although randomized trials support the use of HIPEC in colorectal cancer, ${ }^{15,18-20}$ previous evidence of a beneficial effect of HIPEC in primary ovarian cancer has been limited to single-group trials and retrospective cohorts. ${ }^{12,13}$ In one previous trial involving patients with recurrent ovarian cancer who were randomly assigned to undergo cytoreductive surgery either with or without HIPEC, a significant survival benefit was observed among the patients who received HIPEC. ${ }^{21}$ However, the randomization process was not clearly described, and primary end points were not clearly defined. ${ }^{22}$ Our trial provides data from patients who were randomly assigned to undergo surgery with HIPEC or without HIPEC for the primary treatment of advanced ovarian cancer. Our findings indicate that the addition of HIPEC to complete or optimal interval cytoreductive surgery resulted in longer median recurrence-free survival, by 3.5 months, and longer median overall survival, by 11.8 months, than surgery alone. The effect was consistent across the levels of prespecified stratification factors and other baseline characteristics.

All the patients in our trial received neoadju- vant chemotherapy. Postoperative care was similar in the two trial groups, with the exception of the care that the patients received during the 1-day stay in the ICU after HIPEC that was prespecified in the protocol. The administration of HIPEC had little effect on safety, and the incidence of postoperative complications, the incidence and type of grade 3 or 4 adverse events, and health-related quality-of-life outcomes did not differ significantly between the surgery-plusHIPEC group and the surgery group. The reinitiation of intravenous chemotherapy after surgery was not delayed in either trial group, and no effect of HIPEC on the number of cycles of chemotherapy administered was observed. A single administration of intraperitoneal chemotherapy under hyperthermic conditions differs from repeated postoperative administration of intraperitoneal chemotherapy with respect to pharmacokinetics and pharmacodynamics, which could explain the lower rate of systemic side effects seen with a single administration of intraperitoneal chemotherapy than with a postoperative intravenous or intraperitoneal chemotherapy regimen. ${ }^{17}$ Additional trials are needed to determine 


\begin{tabular}{|c|c|c|c|c|}
\hline \multirow[t]{3}{*}{ Adverse Event } & \multicolumn{2}{|c|}{$\begin{array}{l}\text { Surgery } \\
(N=122)\end{array}$} & \multicolumn{2}{|c|}{$\begin{array}{l}\text { Surgery plus HIPEC } \\
(\mathrm{N}=118)\end{array}$} \\
\hline & Any Grade & Grade 3 or $4 \uparrow$ & Any Grade & Grade 3 or 4 \\
\hline & \multicolumn{4}{|c|}{ number of patients (percent) } \\
\hline Infectiont⿱ & $14(11)$ & $3(2)$ & $21(18)$ & $7(6)$ \\
\hline Abdominal pain & $70(57)$ & $7(6)$ & $71(60)$ & $6(5)$ \\
\hline Ileus & $4(3)$ & $2(2)$ & $9(8)$ & $5(4)$ \\
\hline Pain & $28(23)$ & $2(2)$ & $39(33)$ & $4(3)$ \\
\hline Thromboembolic event $\int$ & $2(2)$ & $2(2)$ & $7(6)$ & $4(3)$ \\
\hline Pulmonary event $\emptyset$ & $8(7)$ & $1(1)$ & $11(9)$ & $3(3)$ \\
\hline Dyspnea & $13(11)$ & 0 & $8(7)$ & $3(3)$ \\
\hline Electrolyte disturbance $\|$ & $6(5)$ & $1(1)$ & $7(6)$ & $3(3)$ \\
\hline Gastrointestinal anastomotic leak & $3(2)$ & $2(2)$ & $3(3)$ & $3(3)$ \\
\hline Nausea & $70(57)$ & $3(2)$ & $74(63)$ & $2(2)$ \\
\hline Fatigue & $37(30)$ & 0 & $44(37)$ & $2(2)$ \\
\hline Cardiac, not otherwise specified & $6(5)$ & $2(2)$ & $8(7)$ & $2(2)$ \\
\hline Neuropathy & $33(27)$ & $1(1)$ & $37(31)$ & $1(1)$ \\
\hline Vomiting & $47(39)$ & $1(1)$ & $32(27)$ & $1(1)$ \\
\hline Anemia & $7(6)$ & $6(5)$ & $5(4)$ & $1(1)$ \\
\hline Pneumonia & $1(1)$ & $1(1)$ & $2(2)$ & $1(1)$ \\
\hline Postoperative hemorrhage & $4(3)$ & $1(1)$ & $2(2)$ & $1(1)$ \\
\hline Hypotension & $11(9)$ & $1(1)$ & $1(1)$ & $1(1)$ \\
\hline Sepsis & $2(2)$ & $2(2)$ & $1(1)$ & $1(1)$ \\
\hline Constipation & $32(26)$ & $1(1)$ & $23(19)$ & 0 \\
\hline Alopecia & $19(16)$ & 0 & $22(19)$ & 0 \\
\hline Diarrhea & $11(9)$ & 0 & $16(14)$ & 0 \\
\hline Fever & $10(8)$ & 0 & $14(12)$ & 0 \\
\hline Dizziness & $15(12)$ & 0 & $9(8)$ & 0 \\
\hline Gastroparesis & $2(2)$ & $2(2)$ & $1(1)$ & 0 \\
\hline Intestinal perforation & $2(2)$ & $2(2)$ & 0 & 0 \\
\hline
\end{tabular}

* Shown are the adverse events of any grade that occurred in at least $10 \%$ of the patients in either trial group, along with all grade 3 or 4 events that occurred in at least two patients. The data from five patients who did not undergo cytoreductive surgery (one patient) or did not receive HIPEC as assigned (four patients) were not included in the analysis of adverse events.

$\uparrow$ In one patient, an event of grade 5 occurred; the patient died after having a colonic perforation that resulted in septic shock.

t Events of infection excluded pneumonia.

$\int$ Thromboembolic events included venous thrombosis, pulmonary embolism, cerebrovascular event, and transient ischemic attack.

If Pulmonary events included hypoxia and respiratory distress.

|| Electrolyte disturbances included hyponatremia, hypernatremia, hypokalemia, hypercalcemia, hypomagnesemia, and hypophosphatemia.

the ways in which HIPEC differs from postoperative intravenous or intraperitoneal chemotherapy and whether HIPEC is also effective after primary cytoreductive surgery.
The overall percentage of bowel resections performed was similar in the two groups, but the percentage of patients who underwent a colostomy or an ileostomy after surgery was significantly 
higher in the surgery-plus-HIPEC group than in the surgery group $(72 \%$ vs. $43 \%, P=0.04)$. Because there is no evidence that HIPEC for ovarian cancer is associated with a higher rate of anastomotic leakage than the rate without HIPEC, this difference in the rate of colostomy or ileostomy could reflect the surgeons' preference.

Randomization in our trial took place at the time of surgery in cases in which complete or optimal cytoreduction was anticipated. The institutional review board at each trial center approved this procedure, which ensured equality of prognosis between the trial groups at the actual time of the trial intervention, although for logistic reasons, randomization was performed before the interval surgery at two of the centers on the basis of the results of a diagnostic laparoscopy that was performed to determine whether complete or optimal surgery was feasible. When HIPEC is added to the surgical treatment, the duration of surgery is extended by 2 hours and a perfusionist is needed. Additional standard costs are incurred owing to the additional 2 hours of surgical time, the disposable products that are needed to administer HIPEC, the use of the HIPEC machine, and the 1-day stay in the ICU.

Our trial involved patients with prognostically unfavorable stage III ovarian cancer who were ineligible for primary cytoreduction owing to extensive abdominal disease. As a result, survival in the control group of our trial was shorter than that in the control group of the Gynecologic Oncology Group (GOG)-172 trial, which included only patients who were eligible for primary cytoreduction. ${ }^{5}$ The recurrence-free survival in our trial was also influenced by the definition of the primary end point, which included elevation of the CA-125 level as determined on the basis of GCIG criteria. When the protocol was designed, measurement of the CA-125 level during follow- up was part of routine clinical practice. However, if the definition of the primary end point had been based on clinical symptoms rather than on measurement of the CA-125 level, the estimated rate of recurrence would have been lower and the absolute prolongation of median recurrencefree survival might have been greater. ${ }^{23}$

The median overall survival was 12 months longer among the patients who received HIPEC than among those who did not receive HIPEC, whereas the median recurrence-free survival was 3.5 months longer with HIPEC than without HIPEC. However, the relative effects of HIPEC on recurrence-free survival and on overall survival were remarkably similar, with hazard ratios of 0.66 for recurrence-free survival and 0.67 for overall survival. The discrepancy between similar relative effects in overall survival and recurrence-free survival and a larger absolute benefit in overall survival than recurrence-free survival reflects the higher rate of disease recurrences than deaths. This finding was also shown in the GOG-172 trial, in which the difference between the trial groups in recurrence-free survival and in overall survival was 5.5 months and 15.9 months, respectively, both in favor of the intraperitoneal chemotherapy group. ${ }^{5}$ The number of patients who received no therapy for recurrent disease in the surgery group was similar to that in the surgery-plus-HIPEC group and cannot explain the difference in absolute benefit between recurrencefree survival and overall survival.

In conclusion, our results indicate that among women with advanced ovarian cancer, HIPEC plus complete or optimal interval cytoreductive surgery resulted in longer survival than cytoreductive surgery alone.

Supported by the Dutch Cancer Society (NKI 2006-4176).

Disclosure forms provided by the authors are available with the full text of this article at NEJM.org.

\section{APPENDIX}

The authors' full names and academic degrees are as follows: Willemien J. van Driel, M.D., Ph.D., Simone N. Koole, M.D., Karolina Sikorska, Ph.D., Jules H. Schagen van Leeuwen, M.D., Ph.D., Henk W.R. Schreuder, M.D., Ph.D., Ralph H.M. Hermans, M.D., Ph.D., Ignace H.J.T. de Hingh, M.D., Ph.D., Jacobus van der Velden, M.D., Ph.D., Henriëtte J. Arts, M.D., Ph.D., Leon F.A.G. Massuger, M.D., Ph.D., Arend G.J. Aalbers, M.D., Victor J. Verwaal, M.D., Ph.D., Jacobien M. Kieffer, Ph.D., Koen K. Van de Vijver, M.D., Ph.D., Harm van Tinteren, Ph.D., Neil K. Aaronson, Ph.D., and Gabe S. Sonke, M.D., Ph.D.

The authors' affiliations are as follows: the Departments of Gynecology (W.J.D., S.N.K.), Biometrics (K.S., H.T.), Surgical Oncology (A.G.J.A.), Pathology (K.K.V.V.), and Medical Oncology (S.N.K., G.S.S.) and the Division of Psychosocial Research and Epidemiology J.M.K., N.K.A.), the Netherlands Cancer Institute, Amsterdam, the Center for Gynecologic Oncology Amsterdam, Amsterdam (W.J.D., S.N.K., J.V.), the Dutch Gynecologic Oncology Group (W.J.D., J.H.S.L., H.W.R.S., R.H.M.H., J.V., H.J.A., L.F.A.G.M., G.S.S.), the Department of Obstetrics and Gynecology, Academic Medical Center, Amsterdam (J.V.), the Department of Obstetrics and Gynecology, Sint Antonius Hospital, Nieuwegein (J.H.S.L.), the Department of Gynecologic Oncology, UMC Utrecht Cancer Center, University Medical Center Utrecht, Utrecht (H.W.R.S.), the Departments of Gynecology and Obstetrics (R.H.M.H.) and Surgery (I.H.J.T.H.), Catharina Hospital, Eindhoven, the Dutch Peritoneal Oncology Group (I.H.J.T.H., A.G.J.A.), the Department of Gynecologic Oncology, 
University of Groningen, University Medical Center Groningen, Groningen (H.J.A.), and the Department of Gynecologic Oncology, Radboud University Medical Center, Nijmegen (L.F.A.G.M.) - all in the Netherlands; and the Department of Surgery, Aarhus University Hospital, Aarhus, Denmark (V.J.V.).

\section{REFERENCES}

1. Rose PG, Nerenstone S, Brady MF, et al. Secondary surgical cytoreduction for advanced ovarian carcinoma. N Engl J Med 2004;351:2489-97.

2. van der Burg MEL, van Lent M, Buyse $M$, et al. The effect of debulking surgery after induction chemotherapy on the prognosis in advanced epithelial ovarian cancer. N Engl J Med 1995;332:629-34.

3. Vergote I, Tropé CG, Amant F, et al. Neoadjuvant chemotherapy or primary surgery in stage IIIC or IV ovarian cancer. N Engl J Med 2010;363:943-53.

4. Wright AA, Bohlke K, Armstrong DK, et al. Neoadjuvant chemotherapy for newly diagnosed, advanced ovarian cancer: Society of Gynecologic Oncology and American Society of Clinical Oncology Clinical Practice Guideline. Gynecol Oncol 2016;143:3-15.

5. Armstrong DK, Bundy B, Wenzel L, et al. Intraperitoneal cisplatin and paclitaxel in ovarian cancer. N Engl J Med 2006;354: 34-43.

6. Tewari D, Java JJ, Salani R, et al. Longterm survival advantage and prognostic factors associated with intraperitoneal chemotherapy treatment in advanced ovarian cancer: a Gynecologic Oncology Group study. J Clin Oncol 2015;33:1460-6.

7. Wright AA, Cronin A, Milne DE, et al. Use and effectiveness of intraperitoneal chemotherapy for treatment of ovarian cancer. J Clin Oncol 2015;33:2841-7.

8. Ohno S, Siddik ZH, Kido Y, Zwelling LA, Bull JMC. Thermal enhancement of drug uptake and DNA adducts as a possible mechanism for the effect of sequencing hyperthermia on cisplatin-induced cytotoxicity in L1210 cells. Cancer Chemother Pharmacol 1994;34:302-6.

9. Panteix G, Beaujard A, Garbit F, et al. Population pharmacokinetics of cisplatin in patients with advanced ovarian cancer during intraperitoneal hyperthermia chemotherapy. Anticancer Res 2002;22(2B): 1329-36.

10. Spratt JS, Adcock RA, Muskovin M Sherrill W, McKeown J. Clinical delivery system for intraperitoneal hyperthermic chemotherapy. Cancer Res 1980;40:256-60. 11. van de Vaart PJ, van der Vange $\mathrm{N}$ Zoetmulder FA, et al. Intraperitoneal cisplatin with regional hyperthermia in advanced ovarian cancer: pharmacokinetics and cisplatin-DNA adduct formation in patients and ovarian cancer cell lines. Eur J Cancer 1998;34:148-54.

12. van Driel WJ, Lok CA, Verwaal V, Sonke GS. The role of hyperthermic intraperitoneal intraoperative chemotherapy in ovarian cancer. Curr Treat Options Oncol 2015;16:14.

13. Cowan RA, O'Cearbhaill RE, Zivanovic O, Chi DS. Current status and future prospects of hyperthermic intraoperative intraperitoneal chemotherapy (HIPEC) clinical trials in ovarian cancer. Int J Hyperthermia 2017:33:548-53.

14. Rustin GJ, Vergote I, Eisenhauer E, et al. Definitions for response and progression in ovarian cancer clinical trials incorporating RECIST 1.1 and CA 125 agreed by the Gynecological Cancer Intergroup (GCIG). Int J Gynecol Cancer 2011; 21:419-23.

15. Verwaal VJ, van Ruth S, de Bree E, et al. Randomized trial of cytoreduction and hyperthermic intraperitoneal chemotherapy versus systemic chemotherapy and palliative surgery in patients with peritoneal carcinomatosis of colorectal cancer J Clin Oncol 2003;21:3737-43.

16. Jaaback K, Johnson N, Lawrie TA. Intraperitoneal chemotherapy for the initial management of primary epithelial ovari- an cancer. Cochrane Database Syst Rev 2016;1:CD005340.

17. Walker JL, Armstrong DK, Huang HQ, et al. Intraperitoneal catheter outcomes in a phase III trial of intravenous versus intraperitoneal chemotherapy in optimal stage III ovarian and primary peritoneal cancer: a Gynecologic Oncology Group study. Gynecol Oncol 2006;100:27-32.

18. Chua TC, Esquivel J, Pelz JOW, Morris DL. Summary of current therapeutic options for peritoneal metastases from colorectal cancer. J Surg Oncol 2013;107: 566-73.

19. Vanounou T, Garfinkle R. Evaluation of cytoreductive surgery and hyperthermic intraperitoneal chemotherapy for peritoneal carcinomatosis of colorectal origin in the era of value-based medicine. Ann Surg Oncol 2016;23:2556-61.

20. Esquivel J. Cytoreductive surgery and hyperthermic intraperitoneal chemotherapy for colorectal cancer: survival outcomes and patient selection. J Gastrointest Oncol 2016;7:72-8.

21. Spiliotis J, Halkia E, Lianos E, et al. Cytoreductive surgery and HIPEC in recurrent epithelial ovarian cancer: a prospective randomized phase III study. Ann Surg Oncol 2015;22:1570-5.

22. Harter P, Reuss A, Sehouli J, Chiva L, du Bois A. Brief report about the role of hyperthermic intraperitoneal chemotherapy in a prospective randomized phase 3 study in recurrent ovarian cancer from Spiliotis et al. Int J Gynecol Cancer 2017; 27:246-7.

23. Rustin GJ, van der Burg ME, Griffin $\mathrm{CL}$, et al. Early versus delayed treatment of relapsed ovarian cancer (MRC OV05/ EORTC 55955): a randomised trial. Lancet 2010;376:1155-63.

Copyright (C) 2018 Massachusetts Medical Society.

\section{TRACK THIS ARTICLE'S IMPACT AND REACH}

Visit the article page at NEJM.org and click on the Metrics tab for a dashboard that logs views, citations, media references, and commentary, with easy linking. Learn more at www.nejm.org/page/article-metrics-faq. 